Document downloaded from:

http://hdl.handle.net/10251/92333

This paper must be cited as:

Ortiz Serna, MP.; Carsí Rosique, M.; Redondo Foj, MB.; Sanchis Sánchez, MJ. (2014). Electrical conductivity of natural rubber cellulose II nanocomposites. Journal of NonCrystalline Solids. 405:180-187. doi:10.1016/j.jnoncrysol.2014.09.026

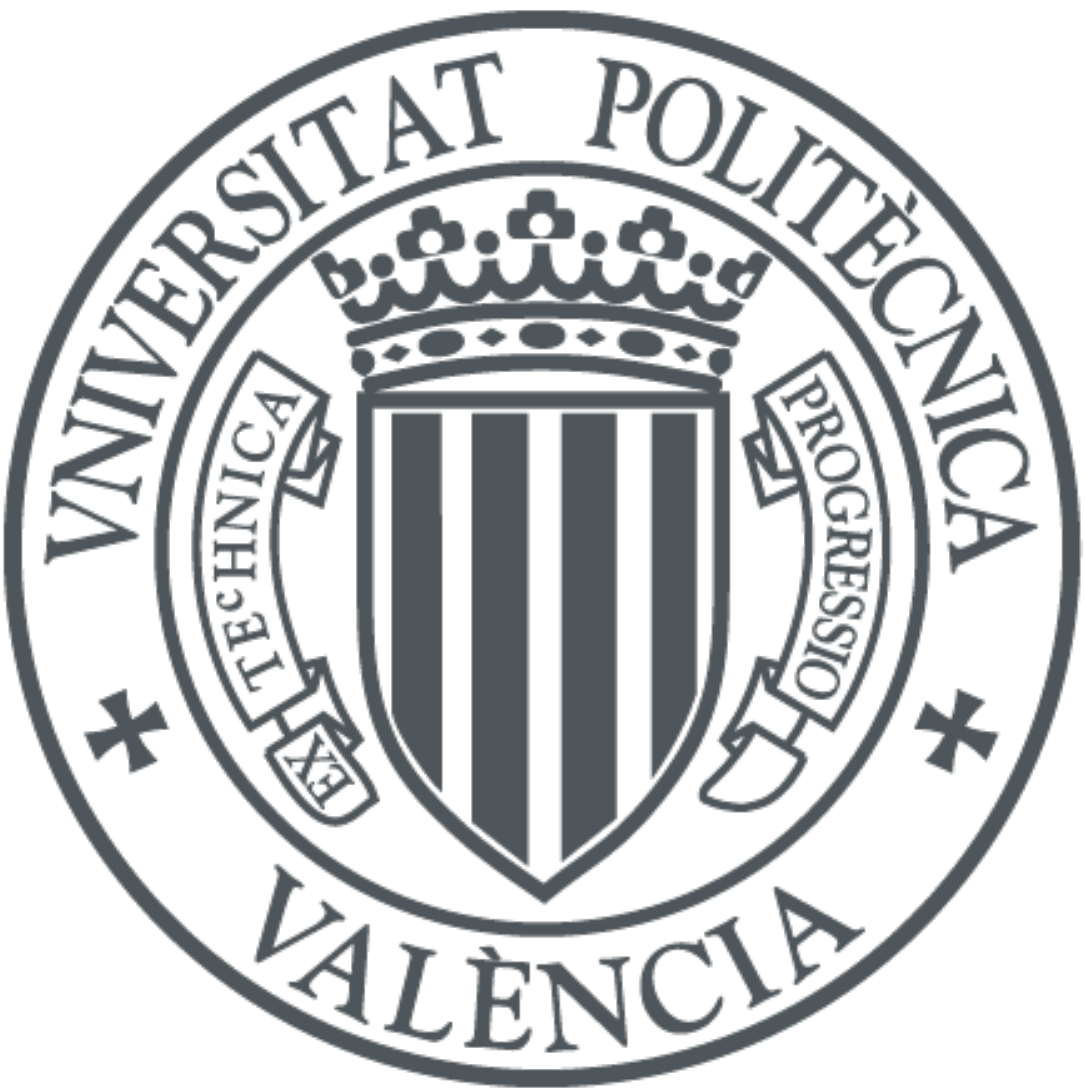

The final publication is available at

http://doi.org/10.1016/j.jnoncrysol.2014.09.026

Copyright Elsevier

Additional Information 


\title{
Electrical conductivity of natural rubber-cellulose II nanocomposites
}

\author{
P. Ortiz-Serna*, M. Carsí, B.Redondo-Foj, M.J. Sanchis \\ Departamento de Termodinámica Aplicada, E.T.S.I.I., Instituto de Tecnología Eléctrica Universitat Politècnica \\ de Valencia, Camino de Vera s/n, 46022 Valencia, Spain
}

* Corresponding author. E-mail address: portiz@ter.upv.es (P. Ortiz-Serna).

\begin{abstract}
Nanocomposite materials obtained from natural rubber (NR) reinforced with different amounts of cellulose II (cell) nanoparticles (in the range of 0 to $30 \mathrm{phr}$ ) are studied by dielectric spectroscopy (DS) in a broad temperature range $\left(-150\right.$ to $\left.150{ }^{\circ} \mathrm{C}\right)$. For comparative purposes, the pure materials, NR and cell, are also investigated. An analysis of the cell content effect on the conductive properties of the nanocomposites was carried out. The dielectric spectra exhibit conductivity phenomena at low frequencies and high temperatures: Maxwell-Wagner-Sillars (MWS) and electrode polarization (EP) conductive processes were observed in the nanocomposite samples.
\end{abstract}

Keywords: Dielectric spectroscopy; Natural rubber; Cellulose II; Nanocomposites; Conductivity

\section{Introduction}

The electrical conductivity of an insulating polymer can be altered by adding conducting particles [1-3]. The conductivity of the composite material can thus be controlled by choosing the suitable components, as shape, size, and relative concentrations [4].

Natural rubber (NR) has been successfully used as an engineering material for many years owing, among other features, to the possibility of compounding it to meet nearly any mechanical requirement and to be electrically insulating or fully conductive. The most common rubber filler in engineering applications is carbon black (CB) $[5,6]$.

All carbon blacks, under normal conditions, have some capability to conduct electricity. However, medium thermal carbon black (ASTM designation of N990) was found to have lower conductivity (higher resistivity) when comparing to general carbon blacks [7]. For that reason medium thermal CB is used, for example, as a functional filler in low voltage wire, cable [8] and automotive radiator hose applications, where the lowest possible level of conductivity is desired. While most carbon blacks are semi-conductive, medium thermal $\mathrm{CB}$ has the highest volume resistivity of all carbon blacks, i.e., is the least suited to conduct electricity in a polymer system. This is due to the fact that in medium thermal $\mathrm{CB}$, individual spheres allow the polymer chains to surround and electrically insulate the carbon. The large particle size and low structure of medium thermal CB are not conducive to the mechanism of semi-conductivity, namely electron tunnelling.

Today, more and more demands are being placed on rubber goods, especially in automotive and aerospace applications. Nevertheless, be- cause of the origin of CB from petroleum this filler causes pollution and gives black colour to rubber. As a result, in the last two decades re- search was focused on 
the development of other reinforcing agents de- rived from inorganic [9,10] and natural organic [11,12] materials to replace carbon black in rubber compounds. It is well known that one of these promising materials is cellulose (cell) [13], which is a renewable raw material, is more environmentally friendly than CB and comes from a sustainable resource. It is for this reason that we have studied a series of NRcell nanocomposite samples in the present work, which is a continuation of previous studies [14-16] concerning the morphological, thermal, mechanical and electrical behaviour of these samples. The morphological characterization showed a good dispersion of cell nanoparticles (with size of $50 \mathrm{~nm}$ or less in diameter) in the composite. Studies on the mechanical and curing properties of these systems showed that cellulose improves the physicochemical as well as technological properties of pure NR. The highest value of the stress at break was achieved for the composite containing $15 \mathrm{phr}$ of cellulose, while the strain at break decreases as the filler content increases above $15 \mathrm{phr}$. The results for the stress and strain at break indicate that the limit of filler content to achieve good mechanical properties is $15 \mathrm{phr}$. Thus, an improvement of the mechanical properties of NR was achieved by the addition of 15 phr of cell while, generally, medium thermal $\mathrm{CB}$ is used in the range of 20 to $50 \mathrm{phr}$ and, moreover, at low loadings of $\mathrm{CB}$, the mechanical properties of the compound will essentially be the same as those without thermal carbon black.

The dielectric spectroscopy technique was used, in the previous work [16], in order to characterize the molecular dynamics of the samples in the low temperature range. However, conduction effects often dominate the dielectric response of a material at high temperatures and low frequencies. The processes that contribute to the dielectric response under these conditions include the migration of mobile charge carriers across the medium and the trapping of charges at interfaces and boundaries. The motion of charge in disordered systems is accompanied by an electrical relaxation, which is characterized by a relaxation time $\tau_{\sigma}$. If the external electric field has a frequency, which is much higher than $1 / \tau_{\sigma}$, its effect on the charge transport is negligible. On the contrary, if $\omega \square 1 / \tau_{\sigma}$ it supports the charge transport and causes a contribution to the electrical relaxation that increases with decreasing frequency. While the motion of charge carriers can increase the dielectric loss by several orders of magnitude, charge trapping influences both, the dielectric permittivity and the loss factor. This additional polarization is the result of (i) the accumulation of charges at the electrode- sample interface, called "electrode polarization" (EP) [4] and/or (ii) the separation of charges at internal phase boundaries referred to as Maxwell-Wagner-Sillars (MWS) polarization [17,18]. MWS polarization is generally evident in non-homogenous materials like multi- phase polymers, blends and colloids, crystalline or liquid crystalline polymers, and composites and occurs across smaller size scales when comparing to the electrode polarization. The MWS effects are more pronounced for conductive materials and, in certain cases, this large-scale polarization can mask the dielectric orientation response of the material.

The aim of the present paper is the phenomenological description and the molecular interpretation of these high temperature relaxation processes found in the solid state, as well as the explanation of the cell nanoparticles and water effect on the nanocomposite conductivity behaviour. In order to characterize the conductive behaviour it is advisable to represent the obtained dielectric data in terms of the complex conductivity $\sigma^{*}(\omega)$. It is remarkable that $\sigma^{*}(\omega)$ is similar in its temperature and frequency dependence for a wide variety of different materials [15-22].

According to Maxwell's equations [23] the current density $\mathrm{j}=\sigma^{*} \mathrm{E}$ and the time derivative of the dielectric displacement $\mathrm{dD} / \mathrm{dt}=\mathrm{i} \omega \varepsilon^{*} \varepsilon_{0} E$ are equivalent, where $\sigma^{*}$ is the complex conductivity, $E$ is 
the electric field, $\varepsilon^{*}$ is the complex permittivity, $\varepsilon_{0}=8.8510^{-12} \mathrm{~F} \cdot \mathrm{m}^{-1}$ is the vacuum permittivity and $\omega=2 \pi \mathrm{f}$ is the angular frequency. Hence, for sinusoidal electrical fields $\mathrm{E}(\omega)=\mathrm{E}_{0} \mathrm{e}^{\mathrm{i} \omega \mathrm{t}}, \varepsilon^{*}(\omega)$ and $\sigma^{*}(\omega)$ are related to each other by $\sigma^{*}(\omega)=\sigma^{\prime}(\omega)+i \sigma^{\prime \prime}(\omega)=i \omega \varepsilon_{0} \varepsilon^{*}(\omega)$. So, the real and imaginary parts of $\sigma^{*}(\omega)$ are given, respectively, by $\sigma^{\prime}(\omega)=\sigma_{\text {ac }}^{\prime}(\omega)=\omega \varepsilon_{0} \varepsilon^{\prime \prime}(\omega)$ and $\sigma^{\prime \prime}(\omega)=\omega \varepsilon_{0} \varepsilon^{\prime}(\omega)$.

We report our experimental results by means of the conductivity dependence on the frequency, temperature, and filler content. Once considered that the cell nanoparticles could be used as a suitable reinforcement in NR composites, these conductivity studies will help to elucidate if NR preserves its inherent insulator behaviour after the cell addition (conductive filler). The results of the morphological characterization pointed out a lack of aggregation of the cell nanoparticles in the NR matrix, and consequently a similar behaviour to that of the medium thermal $\mathrm{CB}$ composites and therefore a feasible use of our samples in the same kind of applications would be expected.

\section{Experimental}

\subsection{Samples}

The nanocomposite materials, labelled as NR10, NR15, NR20, and NR30, consist in four samples in which the cell content varies from 10 to $30 \mathrm{phr}$ (parts per hundred). The syntheses of the samples were carried out in the Instituto de Macromoleculas Professora Eloisa Mano (Universidade Federal do Rio de Janeiro) [24]. These materials were processed to obtain sheets with thickness of around $0.25 \mathrm{~mm}$.

\subsection{Dielectric spectroscopy (DS)}

DS [4,25-27] experiments were performed in a Novocontrol Broad- band Dielectric Spectrometer, based on an Alpha analyser and a Quatro temperature controller. Isothermal measurements were carried out at 44 frequencies between $5 \cdot 10^{-2}$ and $3 \cdot 10^{6} \mathrm{~Hz}$ from -150 to 150 ${ }^{\circ} \mathrm{C}$, in $5{ }^{\circ} \mathrm{C}$ steps, using gold electrodes of $20 \mathrm{~mm}$ in diameter. The accuracy of Alpha impedance measurement is $0.01 \%$. The dielectric measurements were carried out in not only as received but also dried samples labelled respectively as wet and dry from now on. The latter were obtained via drying in an air-circulating oven at $70{ }^{\circ} \mathrm{C}$ until constant weight. The nanocomposites dc conductivity, $\sigma_{\mathrm{dc}}$, was determined from the frequency dependency of the ac conductivity, $\sigma_{\mathrm{ac}}(\omega)$, as the extrapolated value of the conductivity plateau in the low-frequency region.

\section{Results and discussion}

\subsection{Dielectric spectroscopy $(D S)$}

In order to elucidate the influence of the water presence in the molecular dynamic properties of the NR-cell nanocomposites, dielectric measurements were carried out in both wet and dry samples.

Fig. 1 shows the temperature dependence of the dielectric permittivity $\varepsilon^{\prime}$ and loss factor $\varepsilon^{\prime \prime}$ at $1 \mathrm{~Hz}$ for NR, cell II and NR-cell nanocomposites.

As we can observe, several processes are present in the experimental temperature range. In order of increasing temperature, the loss isochrones for all samples present a $\beta$-relaxation, located near $110{ }^{\circ} \mathrm{C}$ and related to the local chain dynamics of the cell II polysaccharide chains $[28,29]$. The high 
temperature side of the $\beta$-process overlaps with the low temperature side of a complex peak, centred near $-60{ }^{\circ} \mathrm{C}$, which can be decomposed into $\alpha$ and $\alpha^{\prime}$-relaxations associated, respectively, with the $T_{g} \mathrm{~s}$ of NR and the lipid component (SA), present in NR not only as a natural impurity but also as an additive [15]. In turn, the high temperature side of the $\alpha$-relaxation overlaps with a process related to the moisture, only detectable in the wet samples. Finally, the increase of the loss factor at high temperatures arises from the conductivity phenomena. Both, ionic conductivity and polarization effects, caused respectively by MWS polarization and EP, are responsible for the dielectric permittivity increase at high temperatures. From the loss factor spectra reported in Fig. 1 it is clear that the intensity of the (i) $\beta$-relaxation, (ii) peak related to the moisture and (iii) conductivity phenomena increases as the cell content rises in the samples. However, the intensity of the complex peak associated with the $\alpha^{\prime}$ and $\alpha$ relaxations is not too much affected by the increase of the cell content. We can also observe an increase of the dielectric permittivity with the cell content. In the case of wet samples, an important increase between 0 and $50^{\circ} \mathrm{C}$ is observed, which can be associated with the water increase in the nanocomposites as the cell content rises. For temperatures above $100{ }^{\circ} \mathrm{C}$, the dielectric permittivity is higher for the dry samples.

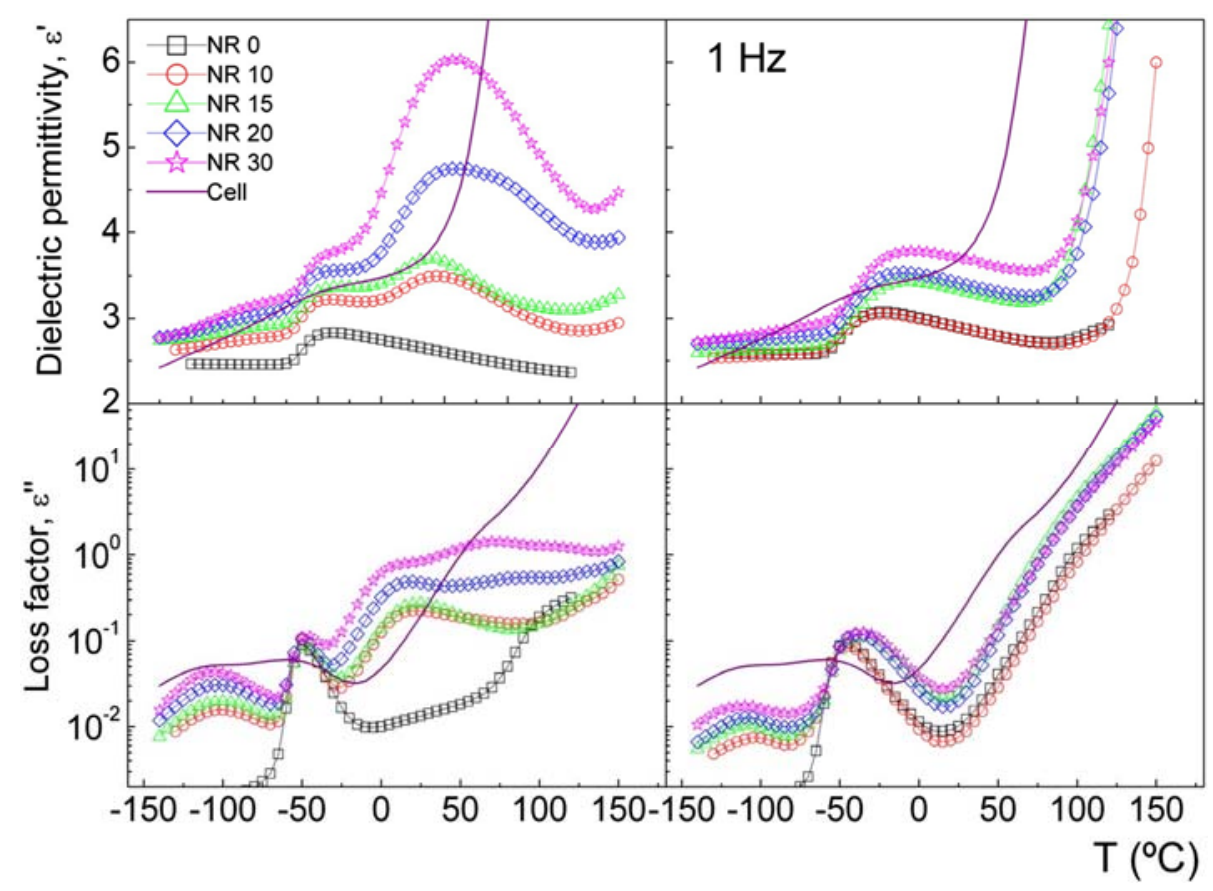

Fig. 1. Temperature dependence of the dielectric permittivity (top) and loss factor (bottom) for wet (left) and dry (right) samples at $1 \mathrm{~Hz}$.

Fig. 2 shows the frequency dependence of the dielectric permittivity at room temperature for the dry NR and NR-cell nanocomposites. The inset displays the variation of the dielectric permittivity with the mass fraction of cell at different frequencies $\left(10^{3}, 10^{2}, 10^{1}\right.$ and $\left.10^{0} \mathrm{~Hz}\right)$. As we can observe in both plots, there is a moderate enhancement of the dielectric permittivity with the cell content. This behaviour is probably related to the increase of the charge carriers blocked at the interfaces between NR and cell, two materials with different electrical behaviours. Clearly, the number of interfaces and hence the charge carriers blocked increases with the cell content in the samples. 
In order to study the dipolar processes, the complex dielectric permittivity was expressed in terms of the Havriliak-Negami (HN) phenomenological equation [30-32]. Assuming an additive rule for the dielectric permittivity [33], the analysis of the loss spectra was carried out for both, wet and dry samples. The parameters that describe the HN equation were computed from the dielectric loss and the pertinent results are collected in our previous work [16].

According to our results, the temperature dependence of the relaxation times of the dry and wet nanocomposites [16] follows the Vogel- Fulcher-Tammann equation [34-36]. Whereas the rate of the $\alpha$-process was unaffected by the water elimination, the slower process $\left(\alpha^{\prime}\right)$ becomes even slower in the dry samples. This fact is related, presumably, to the solvation of the hydrophilic acid groups $\left(-\mathrm{CO}_{2} \mathrm{H}\right)$ present in the lipid component (SA). Thus the water's role is to act as a plasticizer for the lipid process $\left(\alpha^{\prime}\right)$, decreasing the respective glass transition temperature.

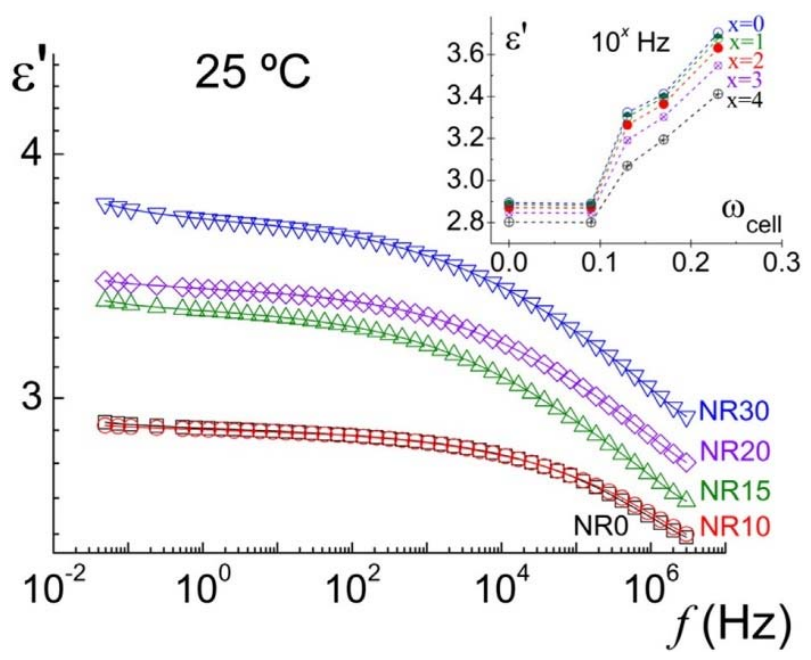

Fig. 2. Frequency dependence of the dielectric permittivity for dry NR and NR-cell nanocomposites at room temperature. Inset shows the variation of permittivity with the mass fraction of cell at several frequencies $\left(10^{x} \mathrm{~Hz}\right.$ with $x=0,1,2,3$ and 4).

For systems in which charge contributions to the dielectric permittivity are important at low frequencies, it is also convenient to analyse the results in terms of the complex dielectric modulus $\left(M^{*}\right)$, a very sensitive parameter to charge transport. Fig. 3 shows the corresponding loss modulus $\left(M^{\prime \prime}\right)$ as a function of temperature for the wet and dry samples.

For both sample series the loss modulus exhibits, in increasing order of temperatures, the relaxation associated with the $\beta$-cell process and the double absorption associated with the NR and SA $T_{g}$ s. The differences between the wet and dry samples are more significant at high temperatures. That is, the wet samples show a peak associated with moisture above the $T_{g}$, which is not present in the dry samples. This peak makes the definition of the MWS and EP processes difficult and for this reason both conductive processes are more clearly defined in the dry samples.

In order to study the effect of the cell content in the conductivity, the frequency dependence of $\sigma^{*}$ for pure polymers and NR-cell nanocomposites, measured at room temperature $\left(20^{\circ} \mathrm{C}\right)$ and at $150{ }^{\circ} \mathrm{C}$ is plotted in Fig. 4. 

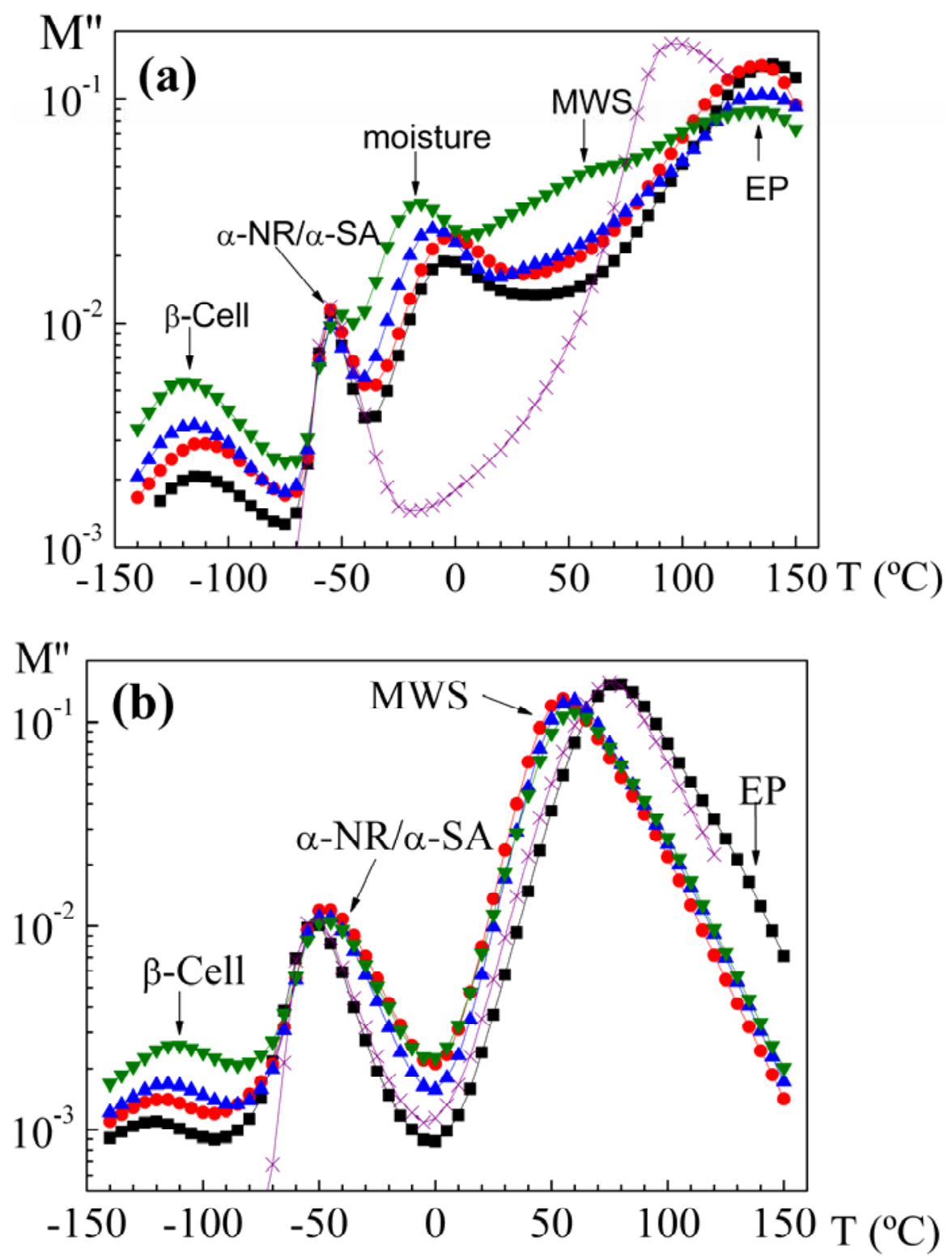

Fig. 3. Temperature dependence of $M^{\prime \prime}$ for (a) wet and (b) dry samples at $0.05 \mathrm{~Hz}$. NR0 (X), NR10 (square), NR15 (circle), NR20 (up triangle) and NR30 (down triangle)

According to Fig. 4, it is evident that the ac conductivity, $\sigma^{\prime}(\omega)$, is both, frequency and temperature dependent, and increases with in- creasing frequency and temperature. However, the influence of the temperature is more pronounced in the low frequency range. At high frequencies all the isotherms collapse. Besides, $\sigma^{\prime}(\omega)$ shows a plateau at high temperature and low frequency range. This plateau represents the $d c$ conductivity of the sample, which increases with growing temperature and cell content, and is clearly marked only for the highest temperatures. As we can observe, in the high frequency range, the frequency dependence of $\sigma^{\prime}(\omega)$ is nearly linear. 

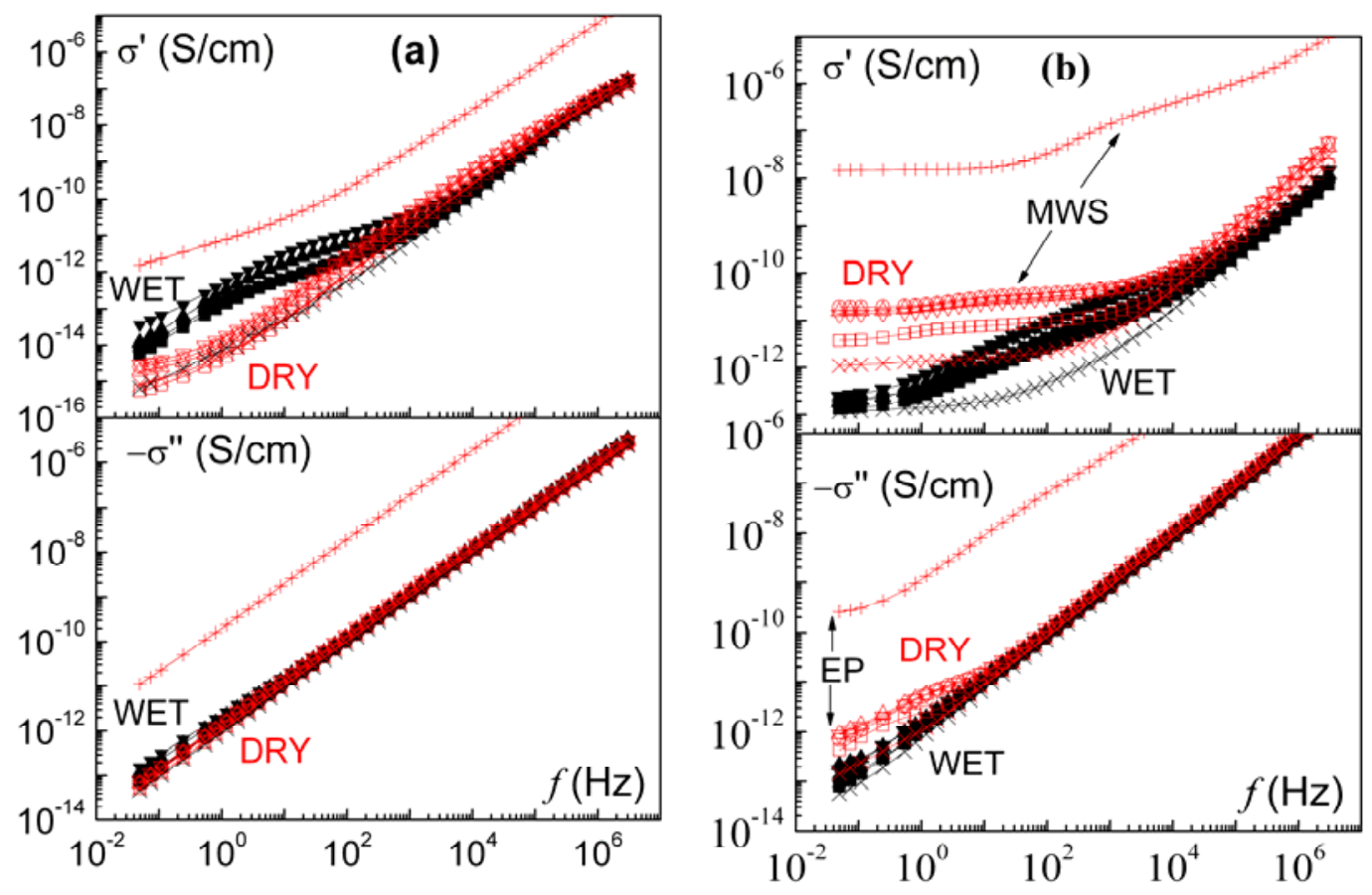

Fig. 4. Frequency dependence of $\sigma^{*}$ for pure polymers and $\mathrm{NR}-$ cell nanocomposites at (a) $20{ }^{\circ} \mathrm{C}$ and (b) $150{ }^{\circ} \mathrm{C}$. NR0 (X), cell (+), NR10 (square), NR15 (circle), NR20 (up triangle) and NR30 (down triangle).

In general the $a c$ conductivity, $\sigma^{\prime}(\omega)$, at a constant temperature can be represented by the following equation

$$
\sigma^{\prime}(\omega)=\sigma_{d c}+A \cdot \omega^{s}
$$

where $\omega$ is the angular frequency, $\sigma_{\mathrm{dc}}$ is the independent frequency conductivity or dc conductivity (at $\omega \rightarrow 0$ ) and the $A$ and $s$ parameters are constants dependent on temperature. The frequency dependence of the conductivity was called by Jonscher as Universal Dynamic Response (UDR) [3741] because a wide variety of materials displayed such behaviour $[39,41]$. The double logarithmic plot of $\sigma^{\prime}$ vs. $\omega$ in the high frequency region follows the power law $\sigma^{\prime}(\omega)=A \omega^{s}$, with $\mathrm{s} \cong 1[42,43]$. This zone is called the nearly constant loss (NLC) regime, because it corresponds to the frequency region in which the dielectric permittivity is nearly independent of frequency. Fig. 5 shows the obtained temperature dependence of the $A$ and $s$ parameters for NR and NR-cell nanocomposites. A multiple linear regression analysis was carried out to calculate the values of both parameters. According to our results, on one hand, when the temperature increases, the exponent $s$ decreases and the $A$ parameter increases. On the other hand, the exponent s increases with the cell content, while non-defined tendency is observed for the $A$ parameter. However, the exponent $\mathrm{s}$ is larger than the universal one $(s=0.8-1)$. This discordance has also been found in some composite systems, comprising of conducting and insulating phases' network, and could probably derive from the fact that the electrical conductivity is achieved by tunnelling, as there are no physical contacts between the fillers. If the cell particles in the NR matrix are not in contact, an activation energy in order to produce the migration of the charge between sites in the whole sample is required. 


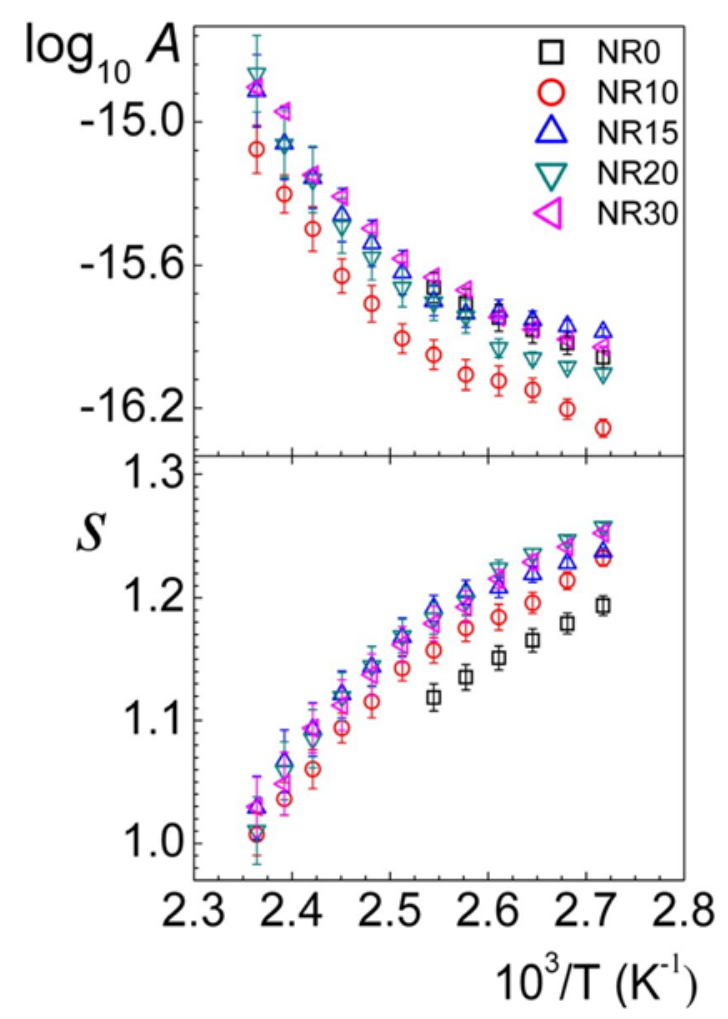

Fig. 5. Temperature dependence of the (a) $A$ and (b) $s$ parameters of the $a c$ conductivity in the high frequency region $\left(\sigma^{\prime}(\omega)=A \omega^{S}\right)$ for NR and NR-cell nanocomposites.

As we can observe in Fig. 4, the bulk conductivity of the pure NR increases with increasing frequency, as expected for an insulator material, with a value at $20^{\circ} \mathrm{C}$ and $10^{-1} \cdot \mathrm{s}^{-1}$ of about $10^{-15} \mathrm{~S} \cdot \mathrm{cm}^{-}$ 1 for both, wet and dry samples. On the other hand, at $150{ }^{\circ} \mathrm{C}$ and $10^{-1} \cdot \mathrm{s}^{-1}$ the value of the conductivity was $10^{-13} \mathrm{~S} \cdot \mathrm{cm}^{-1}$ for the wet samples and $10^{-12} \mathrm{~S} \cdot \mathrm{cm}^{-1}$ for the dry ones. In addition, as explained above, differences in conductivity of the phases of an inhomogeneous medium give rise to interfacial polarization (MWS-relaxation). The charges can migrate under the influence of the applied field contributing to the electrical response of the systems $[1,44]$. Such a polarization usually occurs at frequencies lower than the time scales typical of dipolar polarizations. In the middle part of the isotherm the MWS-relaxation creates a 'knee'- like increase in the $\sigma$-curve, which is uniformly shifted with temperature. At lower temperatures this 'wriggle' is in the range of the $\sigma_{d c}$-plateau, which makes it impossible to determine the $d c$ conductivity directly.

The major effect of even low contents of water is the increase of the electrical conductivity of the sample, which superposes the dielectric processes in the loss spectra [16]. Consequently, it is observed that the MWS process is better defined and more intense in the dry samples than in the wet ones (Fig. 4(b)). This difference can be explained by the changes in the internal interfaces that take place with the water elimination. In accordance with our results, the water elimination would favour the polarization process in the interfaces that exist be- tween the different components of the heterogeneous nanocomposites. As we can observe in Fig. 4, the imaginary part of the complex conductivity decreases with decreasing frequency. The slight increase observed at low frequencies is 
related to the presence of the electrode polarization phenomenon.

For each isotherm, there is a critical frequency $\omega_{c}$ beyond which a power law is followed. The $\omega_{c}$ values were estimated as the frequency at which the $d c$ line intersects with the straight line present in the high frequency zone for each isotherm. Moreover, the value of $\sigma_{d c}$ at each temperature can be estimated from the plateau values of the conductivity in the $\sigma^{\prime}(\omega)-\omega$ plot. The temperature dependence of $\omega_{c}$ and $\sigma_{d c}$ for NR and NR-cell nanocomposites is plotted in Fig. 6(a) and (b) respectively. Both, the plateau value $\sigma_{d c}$ and the critical frequency $\omega_{c}$, decrease with decreasing temperature. Besides, as we can observe in these figures, $\omega_{c}$ and $\sigma_{d c}$ parameters also depend on the cell content. According to our results, the $\omega_{c}$ parameter increases with the cell content until $15 \mathrm{phr}$ and then undergoes a decrease for higher contents of the filler. On the other hand, the $\sigma_{d c}$ parameter increases with the cell content until $15 \mathrm{phr}$ and then remains constant. At low cell concentration (10 phr), the particles are present as individual elements, since the average distance between them exceeds their size, and the conductivity of the composite is very close to that of the pure NR matrix.

In order to analyse both, the effect of the temperature and the composition in the $d c$ conductivity values, Fig. 7 shows the conductivity of the nanocomposites vs. the cell content in the temperature range from 50 to $150{ }^{\circ} \mathrm{C}\left(10^{\circ} \mathrm{C}\right.$ step).

Conductivity increases with the cell content until $15 \mathrm{phr}$, then its value remains approximately constant for higher contents of the filler. The absence of an abrupt increase in the conductivity values with the cell content signifies that all the examined nanocomposite systems exhibit insulator behaviour. However, in Fig. 7 a considerable increase of conductivity with temperature for constant cell content is also observed. As we can see, the $d c$ conductivity values are altered by almost two orders of magnitude with increasing temperature (be- tween 50 and $150{ }^{\circ} \mathrm{C}$ ), which indicates that this is a thermally activated process.

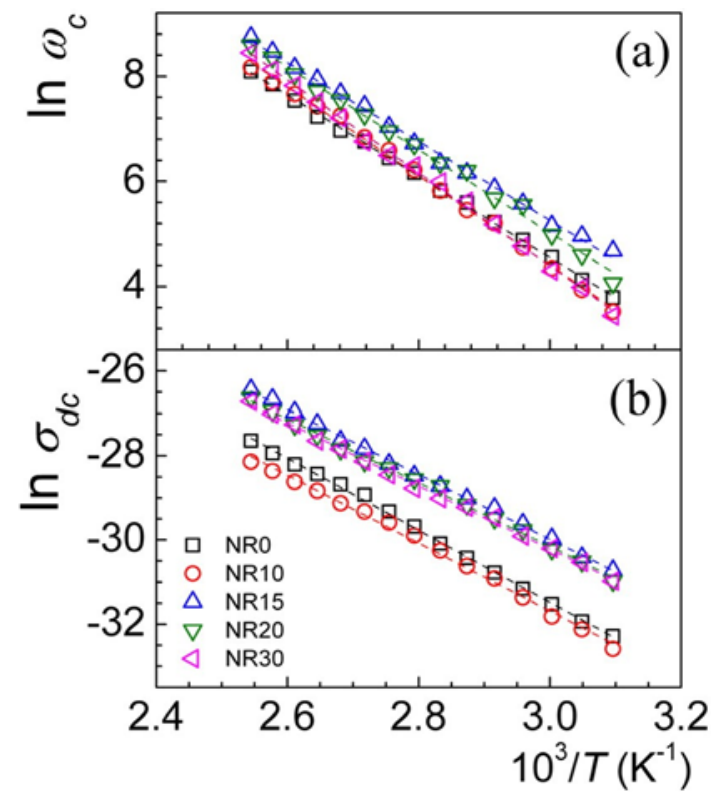

Fig. 6. (a) Temperature dependence of $\omega_{c}(\mathrm{~Hz})$ obtained from the experimental isotherm and (b) temperature dependence of the $d c$ conductivity for the dry samples: NR and NR-cell nanocomposites. All data were satisfactorily linearly fitted. 


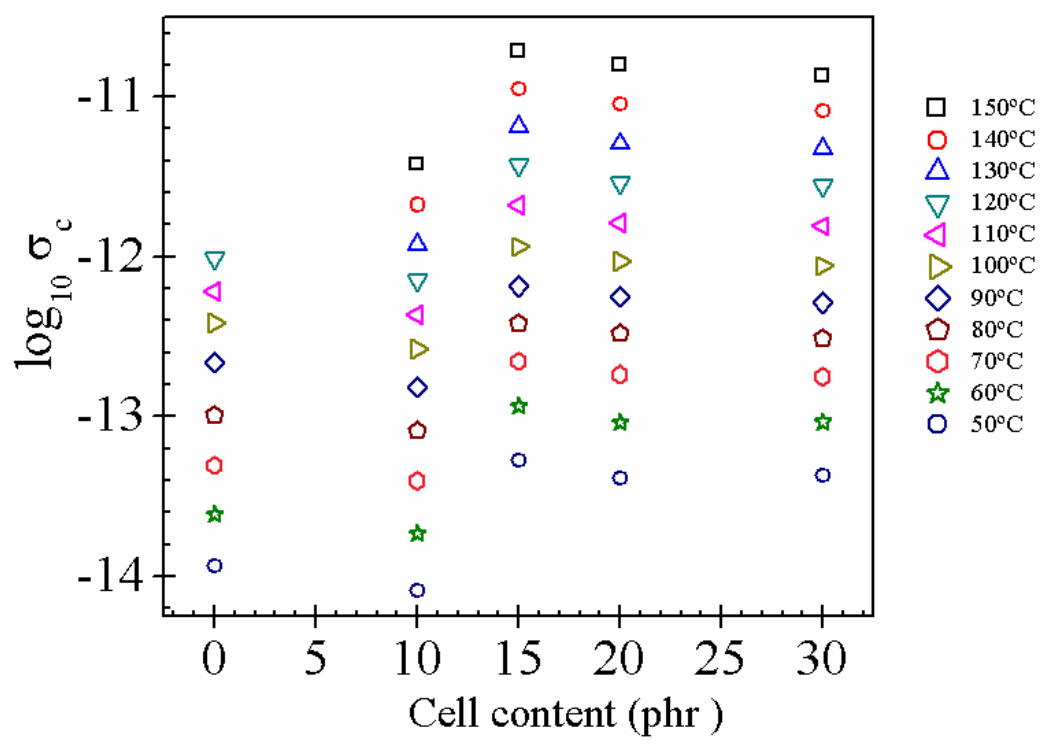

Fig. 7. The $d c$ conductivity $\left(\log _{10} \sigma_{d c}\right)$ versus the cell content of nanocomposites, at several temperatures

The temperature dependence of conductivity can be expressed as follows:

$$
\sigma_{d c}(T)=\sigma_{0} \cdot \exp \left[\frac{E_{a}}{k_{B} T}\right]
$$

where $\sigma_{0}$ is the conductivity at infinite temperature, $E_{a}$ is the activation energy and $k_{B}$ is the Boltzmann constant. The $\sigma_{0}$ parameter can be considered as a constant for each of the examined samples, since the influence of temperature on $\sigma_{0}$ appears to be negligible with respect to the temperature dependence of the exponential factor in Eq. (2). Fig. 6 provides plots of dc conductivity as a function of the reciprocal of temperature for the dry nanocomposites. For the wet samples, the presence of the water process hinders the conductivity evaluation; as a result, the analysis performed for the dried samples was impossible to carry out in this case. As we can observe in Fig. 6, the experimental data were satisfactorily linearly fitted, revealing the Arrhenius type or the single thermally activated process. From the linear fits the activation energy and the $\sigma_{0}$ parameter were evaluated and the obtained values are summarized in Table $\mathbf{1}$ for the dry samples.

Table 1. Values of the fit parameters of Eq. (2) for the dry NR and NR-cell nanocomposites

\begin{tabular}{|c|c|c|c|c|c|}
\hline & \multirow[t]{2}{*}{$\begin{array}{l}\text { Cell } \\
(\mathrm{Phr})\end{array}$} & \multicolumn{2}{|c|}{$\begin{array}{l}\text { From } \sigma_{d c} \text { obtained with the } \\
\text { plateau of } \sigma^{\prime}(\omega \rightarrow 0)\end{array}$} & \multicolumn{2}{|c|}{$\begin{array}{l}\text { From } \sigma_{d c} \text { obtained with fit to } \\
\text { eq. (5) }\end{array}$} \\
\hline & & $\ln \sigma_{0}\left[\mathrm{~S} \cdot \mathrm{cm}^{-1}\right]$ & $E_{a}\left(\mathrm{~kJ} \cdot \mathrm{mol}^{-1}\right)$ & $\ln \sigma_{0}\left[\mathrm{~S} \cdot \mathrm{cm}^{-1}\right]$ & $E_{a}\left(\mathrm{~kJ} \cdot \mathrm{mol}^{-1}\right)$ \\
\hline NR0 & 0 & $-5.8 \pm 0.2$ & $71.2 \pm 0.7$ & $-6.5 \pm 0.3$ & $68.5 \pm 0.9$ \\
\hline NR10 & 10 & $-7.5 \pm 0.4$ & $67.1 \pm 1.2$ & $-7.1 \pm 0.3$ & $67.5 \pm 0.9$ \\
\hline NR15 & 15 & $-6.9 \pm 0.3$ & $63.9 \pm 0.8$ & $-6.1 \pm 0.4$ & $65.6 \pm 1.2$ \\
\hline NR20 & 20 & $-7.5 \pm 0.3$ & $62.8 \pm 1.0$ & $-6.8 \pm 0.1$ & $64.3 \pm 0.3$ \\
\hline NR30 & 30 & $-7.8 \pm 0.3$ & $62.2 \pm 0.8$ & $-7.0 \pm 0.2$ & $63.6 \pm 0.7$ \\
\hline
\end{tabular}


A slight decrease in the values of activation energy and $\sigma_{0}$ parameter with the cell content is observed although non-relevant changes are produced. So, the values of the activation energy for the $\sigma$-process are similar for all the samples and lie between 62 and $71 \mathrm{~kJ} \cdot \mathrm{mol}^{-1}$. The activation energy reflects the microstructure of the composites, thus, the reduction of inter-particle separation by increasing the volume fraction of the cell phase could be responsible for the slight decrease in the values of the activation energy.

A general approach for the study of the time/frequency temperature correspondence for the $a c$ conductivity is to use the scaling ansatz $[45,46]$

$$
\sigma^{\prime}(\omega, T)=\sigma_{d c} \cdot f\left(\frac{\omega}{\omega_{c}(T)}\right)=\sigma_{d c}\left[1+\left(\frac{\omega}{\omega_{c}}\right)^{t}\right]
$$

where $f\left(\omega / \omega_{c}\right)$ is the so-called scaling function and $\omega_{c}$ the previously de- fined angular frequency marking the onset of the $a c$ conductivity. Scaling properties are shown in Fig. 8 where the same data have been redrawn in reduced units, and the reduced conductivity $\sigma^{\prime}(\omega, T) / \sigma_{d c}(T)$ is plotted against the reduced frequency $\omega / \omega_{c}(T)$. The results of Fig. 8 show that this scaling law holds not only for disordered ion conducting inorganic systems but also for polar viscoelastic liquids.
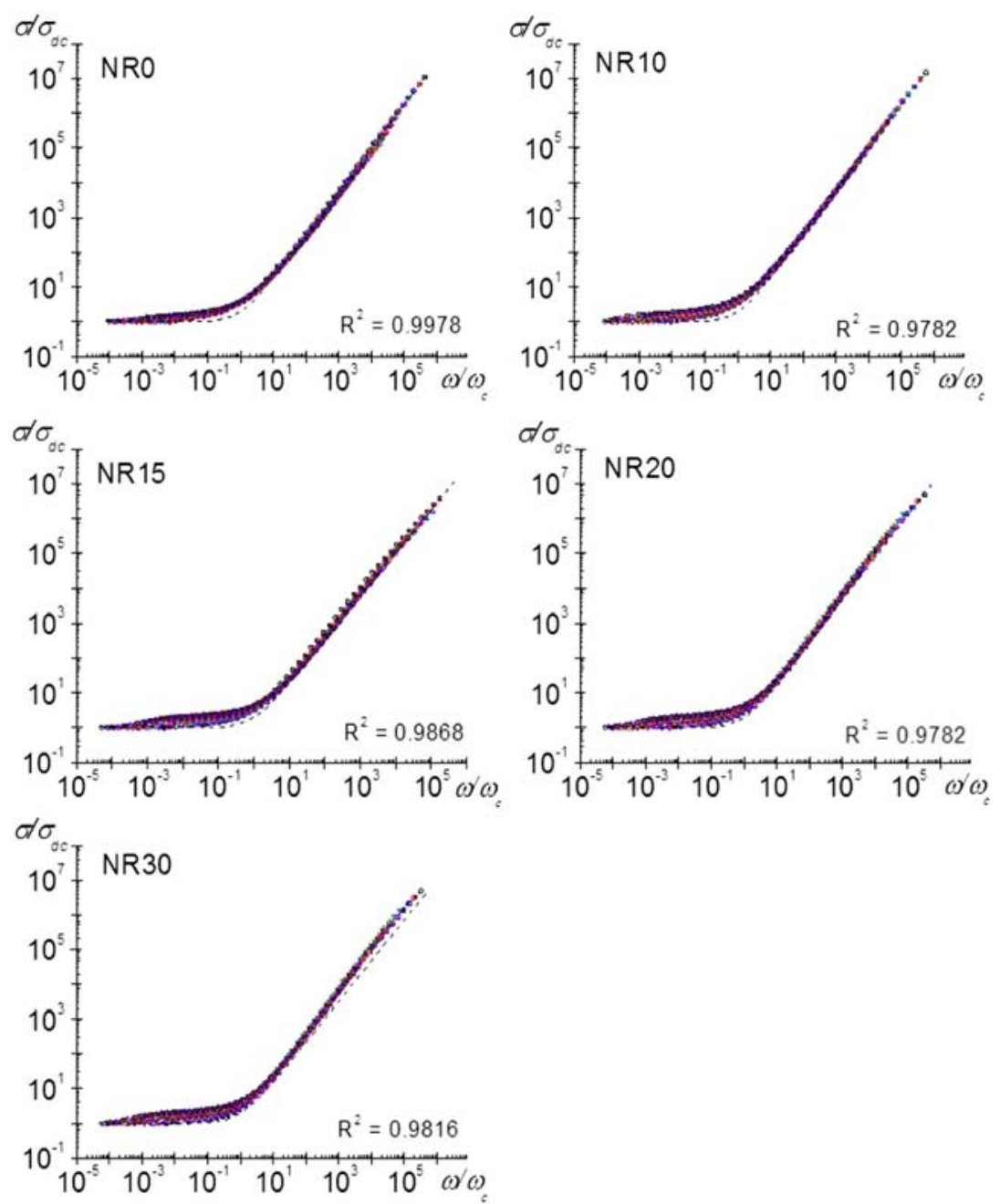

Fig. 8. Frequency dependence scaling spectra for the $a c$ conductivity using the scaling ansatz $\sigma^{\prime}(\omega, T)=\sigma_{d c} f\left[\omega / \omega_{c}(T)\right]$ (from 50 to $120^{\circ} \mathrm{C}, 5^{\circ} \mathrm{C}$ step). 
As we can see, the superposition is better in the high frequency range, whereas the overlapping fails in the MWS zone. The data were fitted to Eq. (3) by multiple regression analysis with a single value of $t$ for different $T: t=1.2500 \pm 0.0001$ for NR0, $t=1.2529 \pm 0.0005$ for NR $10, t=1.2527 \pm 0.0004$ for NR15, $t=1.2229 \pm 0.0005$ for NR20 and $t=1.1744 \pm 0.0004$ for NR30.

Various theoretical models [47] for $a c$ conductivity have been predicted in order to explain the experimental observations of complex systems. So, to examine the electrical transport properties of the matrix-filler systems, different hopping models have been proposed, as for example, the variable range hopping model [48,49] and the random free-energy barrier model [40,41,50-52]. The term hopping refers to sudden displacement of the charge carrier from one position to another neighbouring, and in general includes both, jumps over a potential barrier and quantum mechanical tunnelling [37].

Among several models proposed for the interpretation of the charge carrier transport mechanism, the simplest is the random free-energy barrier model (also referred to as the symmetric hopping model) proposed by Dyre [41,50]. This model is based on the ascertainment that the $d c$ conductivity is thermally activated (Eq. (2)), and the $a c$ conductivity is less temperature dependent. The latter suggests that processes with activation energies smaller than $E^{\sigma d c}$ dominate the $a c$ conductivity. In our case the experimental data are satisfactorily linearly fitted to Eq. (2), revealing that it is a single thermally activated process. According to this model the non-interacting charge carriers remain at sites with minimum energy. The charge carrier transport mechanism is produced when the carriers acquire enough energy to overcome the energy barrier needed to jump to the nearest neighbour site. From the above assumptions and employing a continuous time random walk approximation [53], Dyre has derived the following equation for the $a c$ conductivity in disorder solids [41]

$$
\sigma_{a c}^{*}(\omega)=\sigma_{d c}\left[\frac{j \omega \tau_{e}}{\ln \left(1+j \omega \tau_{e}\right)}\right]
$$

where $\tau_{e}$ is the time involved in overcoming the highest barrier that determines the conductivity and $\sigma_{d c}$ the $d c$ conductivity. This model has been found to be in agreement with experimental data for a large number of disordered solids [40,41]. Taking into account that $\left(1+j \omega \tau_{e}\right)=(1+$ $\left.\omega^{2} \tau_{e}^{2}\right)^{1 / 2} e^{j \arctan \left(\omega \tau_{e}\right)}$, thereal and imaginary components of $\sigma^{*}$ are given by

$$
\begin{aligned}
& \sigma^{\prime}(\omega)=\sigma_{a c}=\frac{\sigma_{d c} \cdot \omega \cdot \tau_{e} \arctan \left(\omega \tau_{e}\right)}{(1 / 4) \ln \left(1+\omega^{2} \tau_{e}^{2}\right)+\left[\arctan \left(\omega \tau_{e}\right)\right]^{2}} \\
& \sigma^{\prime \prime}(\omega)=\frac{\sigma_{d c} \cdot \omega \cdot \tau_{e} \ln \left(1+\omega^{2} \tau_{e}^{2}\right)}{(1 / 2) \ln \left(1+\omega^{2} \tau_{e}^{2}\right)+2\left[\arctan \left(\omega \tau_{e}\right)\right]^{2}} .
\end{aligned}
$$

Fig. 9(a) and (b) shows the temperature dependence of the $\sigma_{d c}$ and $\tau_{e}$ parameters obtained from the fits to Eq. (5a). This dependence is Arrhenius like and the obtained activation energies are between 64 and $68 \mathrm{~kJ} \cdot \mathrm{mol}^{-1}$ (see values in Table 1). These values are in agreement with the one evaluated from the $\sigma_{d c}$ obtained from the plateau in Fig. 4(b). 
Moreover, the plot $\sigma_{\mathrm{dc}} v s . \tau_{\mathrm{e}}{ }^{-1}$ proves howwell the BNN relationship [54-56] is fulfilled by the data independently of the cell content (see Fig. 9(c)). This behaviour suggests that both, dc conductivity and ac conductivity are based on the same mechanism of charge transport.

\section{Conclusions}

This work is mainly focused on the electrical properties of a series of NR matrix loaded with different amounts of cell nanoparticles. The DS technique was employed to study the cell nanoparticles and water con- tent effect on the nanocomposite conductivity behaviour.

It was found that interactions between the cell particles and the NR matrix slightly influence the electrical conductivity of the NR-cell nanocomposites.

Wet nanocomposites present higher conductivity than NR0, and be- come more conductive as the cell content rises, due to the increase of water and polar groups in the samples. In addition, the charge carriers blocked at interfaces due to the MWS effects increases with the cell content due to the rise of internal interfaces. Accordingly, we observe a slight increase of the dielectric permittivity at room temperature, while larger increases are observed at higher temperatures, as expected.

As well as for the wet samples, MWS and EP processes are observed in the dry nanocomposites and the samples also present higher conductivity than NR0. In the dry samples, the conductivity increases until $15 \mathrm{phr}$ is reached and then becomes more or less constant. According to our results, the conductivity of the nanocomposites is limited by the conductivity of the NR matrix in both, wet and dry samples and the contribution of cell conductivity is not enough to modify the insulating behaviour of these materials. For dry nanocomposites, the $d c$ conductivity values, $\sigma d c$, were used to obtain the activation energy for the $\sigma$-process. The reduction of inter-particle separation by increasing the volume fraction of the cell phase results in a decrease of the corresponding values of the activation energy. This is also supported by the obtained $s$ and $t$ values for the Jonscher and scaling ansatz equations, which are an indication that there is no physical contact between the cell particles inside the NR matrix, thus hindering the electron tunnelling mechanism.

Cell nanoparticles maintain the inherent good dynamic properties of NR. Consequently, there is potential for this filler to provide loaded NR compounds having good processing and physical properties, without sacrificing the insulating properties in applications where the lowest possible level of conductivity is desired. These findings may be useful as a guideline for the development of rubber compounds for engineering purposes with required performance.

\section{Acknowledgements}

We thank Professor Regina Nunes of the Instituto de Macromoléculas Eloisa Mano (Universidade Federal do Rio de Janeiro) for providing us the NR and NR-cell samples. This work was financially supported by DGCYT through grant MAT2012-33483. 


\section{References}

[1] G.C. Psarras, E. Manolakaki, G.M. Tsangaris, Compos. Part A 33 (2002) 375.

[2] Y. Mamunya, V.V. Davydenko, P. Pissis, E.V. Lebedev, Eur. Polym. J. 38 (9) (2002) 887.

[3] S. Barrau, P. Demont, A. Peigney, C. Laurent, C. Lacabanne, Macromolecules 36 (2003) 5187.

[4] F. Kremer, A. Schönhals, Broadband Dielectric Spectroscopy, Springer-Verlag Berlin, Heidelberg New York, 2003.

[5] J. Donnet, Carbon Black: Physics, Chemistry and Elastomer Reinforcements, Marcel Dekker, Inc., New York, 1976.

[6] E.J. Queen, Carbon black, in: H.S. Katz, J.S. Milewski (Eds.), Handbook of Fillers and Reinforcements for Plastics, Van Nostrand Reinhold Company, 1978.

[7] J.B. Donnet, Carbon Black: Science and Technology, 2nd ed. CRC Press, 1993.

[8] J.D. Hogan, "Wire and cable" in the Vanderbilt Rubber Handbook, 13th ed., 1990. 717.

[9] M. Arroyo, M.A. López-Machado, B. Herrero, Polymer 44 (2003) 2447-2453.

[10] A.P. Meera, S. Said, J. Phys. Chem. C 113 (2009) 17997-18002.

[11] H. Angellier, S. Molina-Boisseau, A. Dufresne, Macromolecules 38 (2005) 9161-9170.

[12] K. Gopalan, A. Dufresne, Biomacromolecules 4 (2003) 657-665.

[13] Jaehwan Kim, Sungryul Yun, Zoubeida Ounaies, Macromolecules 39 (2006) 4202-4206.

[14] R.C. Reis-Nunes, V. Compañ, E. Riande, J. Polym. Sci. B Polym. Phys. 38 (2000) 393-402.

[15] P. Ortiz-Serna, R. Díaz-Calleja, M.J. Sanchis, G. Floudas, R. Nunes, Macromolecules 43 (2010) 5094.

[16] P. Ortiz-Serna, R. Díaz-Calleja, M.J. Sanchis, E. Riande, R. Nunes, J. Non-Cryst. Solids 357 (2011) 598.

[17] K.W. Wagner, Arch. Electrotech. 2 (1914) 371.

[18] R.W. Sillars, Inst. Electr. Eng. 80 (1937) 378.

[19] C.A. Angell, Chem. Rev. 90 (1990) 523.

[20] P. Köberle, A. Laschewsky, Macromolecules 27 (1994) 2165.

[21] A.J. Epstein, AC conductivity of polyacetylene: distinguishing of charge transport, in:

T.A. Skotheim (Ed.), Handbook of Conducting Polymers, vol. 2, Marcell Dekker, New York, 1986, p. 1041.

[22] M.T. Connor, S. Roy, T.A. Ezquerra, F.J. Baltá-Calleja, Phys. Rev. B 57 (1998) 2286. [23] J.C. Maxwell, Phil. Trans. 155 (1865) 459.

[24] A.F. Martins, L.L.Y. Visconte, R.C.R. Nunes, Kautschuk Gummi Kunststoffe 55 (12) (2002) 637. 
[25] N.G. McCrum, B.E. Read, W. Williams, Anelastic and Dielectric Effects in Polymeric Solids, Dover Publications, Inc., New York, 1991.

[26] E. Riande, R. Díaz Calleja, Electrical Properties of Polymers, Marcel Dekker Inc., 2004 [27] A.K. Jonscher, Dielectric Relaxation in Solids, Chelsea Dielectric press, 1983.

[28] J. Einfeldt, et al., Prog. Polym. Sci. 26 (2001) 1419.

[29] D. Meißner, J. Einfeldt, A. Kwasniewski, J. Non-Cryst. Solids 275 (2000) 199.

[30] S. Havriliak, S.J. Havriliak, Dielectric and Mechanical Relaxation in Materials, Hanser, Munich, 1997.

[31] S. Havriliak, S. Negami, J. Polym. Sci. C Polym. Symp. 14 (1966) 99. [32] S. Havriliak, S. Negami, Polymer 8 (4) (1967) 161.

[33] E. Donth, J. Polym. Sci. B Polym. Phys. 34 (17) (1996) 2881.

[34] H. Vogel, Z. Phys. 22 (1921) 645.

[35] G.S. Fulcher, J. Am. Ceram. Soc. 8 (1925) 339.

[36] G. Tamman, W. Hesse, Z. Anorg. Allg. Chem. 156 (1926) 245.

[37] G.C. Psarras, Compos. Part A 37 (2006) 1545.

[38] H. Böttger, U.V. Bryskin, Hopping Conduction in Solids, Verlag Akademie, Berlin, 1985.

[39] A.K. Jonscher, Nature 267 (1977) 673.

[40] A.K. Jonscher, Universal Relaxation Law, Chelsea Dielectrics Press, London, 1992. (Chap. 5).

[41] J.C. Dyre, T.B. Schroder, Rev. Mod. Phys. 72 (2000) 873.

[42] J.C. Dyre, J. Appl. Phys. 64 (5) (1988) 2456.

[43] S.R. Elliott, Adv. Phys. 36 (1987) 135.

[44] A. Burns, G.D. Chryssikos, E. Tombari, R.H. Cole, W.M. Risen, Phys. Chem. Glasses 30 (1989) 264.

[45] K.L. Ngai, J. Chem. Phys. 110 (1999) 10576.

[46] R. Murugaraj, J. Mater. Sci. 42 (2007) 10065.

[47] A.N. Papathanassiou, I. Sakellis, J. Grammatikakis, Appl. Phys. Lett. 91 (2007) 122911.

[48] N.F. Mott, Metal Insulator Transitions, Taylor \& Francis, London, 1990.

[49] N.F. Mott, Conduction in Non-crystalline Materials, Clarendon Press, Oxford, 1987. [50] J.C.

Dyre, J. Phys. C Solid State Phys. 19 (1986) 5655.

[51] J.C. Dyre, T.B. Shrøder, Phys. Status Solidi B 230 (1) (2002) 5.

[52] T.B. Shrøder, J.C. Dyre, Phys. Chem. Chem. Phys. 4 (2002) 3173.

[53] E. Montroll, G.H. Weiss, J. Math. Phys. 6 (1965) 167.

[54] J.L. Baton, Verres Refract. 20 (1966) 328. 
[55] H. Nakajima, Annual Report of Conference on Electric Insulation and Dielectric Phe nomena, National Academy of Sciences, Washington D.C., 1971

[56] H. Namikawa, J. Non-Cryst. Solids 18 (1975) 173. 